\title{
Mathematical Modelling in 3D of Opener with Scatterer of the Grain-Fertilizer Seeder
}

\author{
Sayakhat NUKESHEV*, Valentin SLAVOV**, Nurbol KAKABAYEV***, \\ Maxat AMANTAYEV***** \\ *S.Seifullin Kazakh Agro Technical University, Pobedy 62, 010011 Astana, Kazakhstan, E-mail: snukehev@mail.ru \\ **University of Chemical Technology and Metallurgy, Sv. Kliment Ohridski Bull., 1756 Sofia, Bulgaria, \\ E-mail:valslavov@abv.bg \\ ***Sh.Ualikhanov Kokshetau State University, Abaya 76, 020000, Kokshetau, Kazakhstan, E-mail: nurbol.ka@mail.ru \\ ****Kostanay department of "Kazakh Scientific Research Institute of Mechanization and Electrification of Agriculture" \\ LLP, Abay Ave., 34,010011, Kostanay,Kazakhstan,E-mail: amantaevmaxat.kz@mail.ru \\ cross ref http://dx.doi.org/10.5755/j01.mech.24.6.22476
}

\section{Introduction}

One of the ways of increasing the yield of crops is the applying the subsoil scattered sowing of grain crops, which is allowed to optimally use of the feeding area by the crop plants and provide the utilization of $70-100 \%$ of the field area at the scattered sowing [1-4].

In this regard, an experimental model of the seeding opener of the grain-fertilizer seeder with the scatterer for subsoil scattered sowing of seeds was developed [5].

The offered seeding opener contains a shank, seed delivery tube and sweep tine furrow opener. The sweep tine furrow opener with a visor form a closed subsoil space for uniformly seeding over the whole seedbed area. Inside, under the sweep tine furrow opener space the oscillating scatterer in the form of a pendulum hemisphere is hanged by a pin, guide bracket and rob. The scatterer-pendulum hemisphere is full volume, all-metal and on the horizontal plane has two degrees of freedom.

The sowing process occurs as follows. During the falling of the seed mass, they are hit to around the oscillating scatterer-pendulum hemisphere and are scattered in all directions, are uniformly being distributed over the entire width of the sweep tine furrow opener.

The process of seeds scattering and distribution by different types of scatterers of seeding openers of the grain-fertilizer seeders was studied by many researchers, namely, A. P. Svevchenko, V. A. Domrachev, E. V. Krasilnikov, N. M. Bespamyatnova, P. V. Lavrukhin and etc. [6-9]. However, the scatterer in the form of pendulum hemisphere has not been drawn a research attention. In doing so, modelling of the different mechanical systems by the matrix methods has been done by numerous researchers, in particular, J. Denavit, R.S. Hartenberg, J.J. Uicker, I. Angelov, V. Slavov, F.M.L. Amiroche and etc. [10-13].

Hence, the purpose of these studies is the mathematical modelling in 3D space of the developed oscillating scatterer-pendulum hemisphere of seeding opener of the grain-fertilizer seeder using the matrix method.

\section{Materials and methods}

For the mathematical modelling have been used the matrix method. The investigated model is presented as a mechanical system with eight degrees of freedom. The calculation procedure has been done in the computing mathematical program Wollframm.

To describe the motion of seeding opener with the oscillating scatterer of the grain-fertilizer seeder a kinematic model of the investigated mechanical system by using the following designations is considered (Fig. 1):

$O_{0} X_{0} Y_{0} Z_{0}$ - initial rectangular coordinate system;

$O_{1} X_{1} Y_{1} Z_{1}$ - rectangular coordinate system connected with the frame of seeder (body 1 );

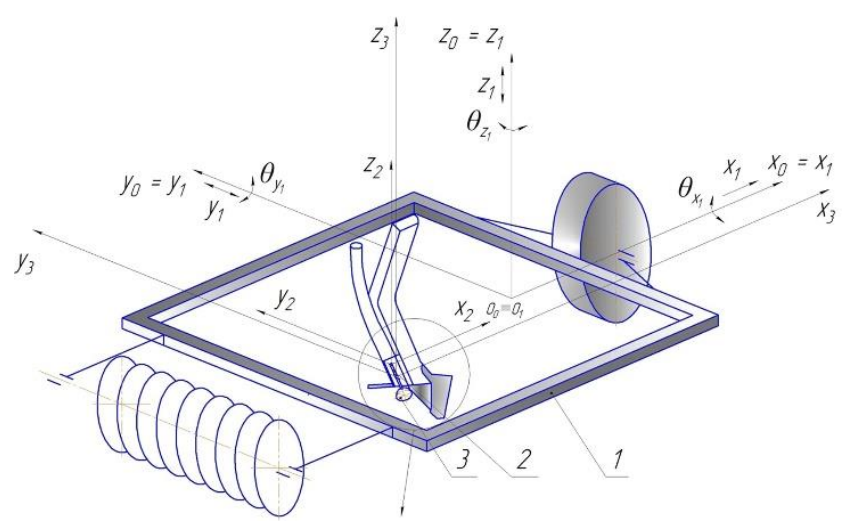

a

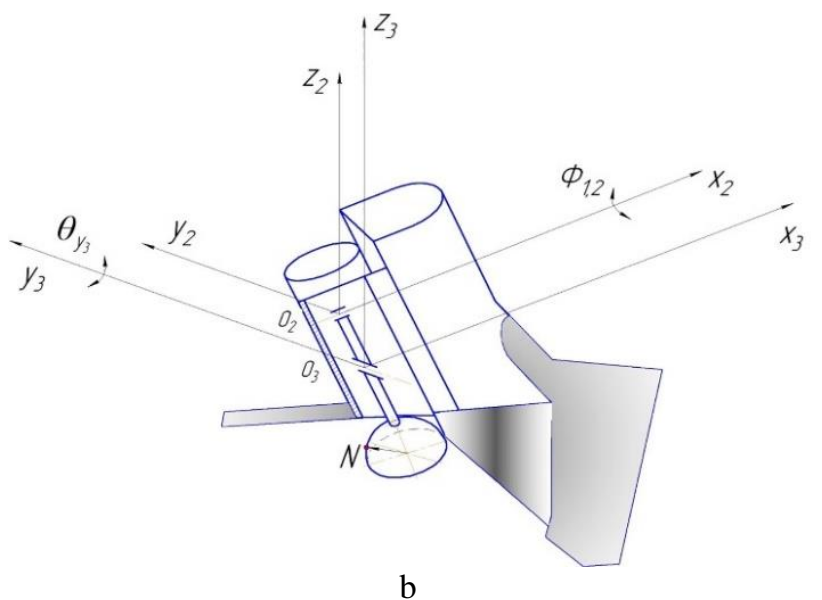

Fig. 1 Kinematic model in 3D space: a - the mechanical system of the units of the grain-fertilizer seeder; $\mathrm{b}$ - the seeding opener with the oscillating scatterer

$\mathrm{O}_{2} \mathrm{X}_{2} \mathrm{Y}_{2} \mathrm{Z}_{2}$ - rectangular coordinate system connected with the guide bracket (body 2 ); 
$\mathrm{O}_{3} \mathrm{X}_{3} Y_{3} \mathrm{Z}_{3}$ - rectangular coordinate system connected with the oscillating scatterer-pendulum hemisphere (body 3);

$x_{1}, y_{1}, z_{1}-$ a small movements of the frame of seeder along the coordinate axes (body 1 );

$\theta_{x 1}, \theta_{y 1}, \theta_{z 1}-$ a small rotation of the frame of seeder around the coordinate axes (body 1 );

$\Phi_{12}$ - degree of freedom - rotation of guide bracket around the axis $\mathrm{O}_{2} X_{2}$ (body 2);

$\theta_{y 3}$ - degree of freedom - rotation of the oscillating scatterer-pendulum hemisphere around the axis $\mathrm{O}_{3} \mathrm{Y}_{3}$ (body 3);

$N_{1}, N_{2}, N_{3}$ - the considered points on the oscillating scatterer-pendulum hemisphere.

\section{Research results}

The vector of the generalized coordinates of the mechanical systems is written as follows:

$$
\mathbf{q}=\left[\begin{array}{llllllll}
x_{1} & y_{1} & z_{1} & \theta_{x 1} & \theta_{y 1} & \theta_{z 1} & \Phi_{12} & \theta_{y 3}
\end{array}\right]_{8 \times 1}^{T} .
$$
For body 1:

The transition matrix between coordinate systems:

- small transitions by coordinates:

$$
\mathbf{A t}_{1}^{0}=\left[\begin{array}{cccc}
1 & 0 & 0 & x_{1} \\
0 & 1 & 0 & y_{1} \\
0 & 0 & 1 & z_{1} \\
0 & 0 & 0 & 1
\end{array}\right]
$$

- a small rotation around the $O_{1} X_{1} Y_{1} Z_{1}$ coordinate system axis:

$$
\begin{aligned}
\mathbf{A u}_{\mathbf{x} 1} & =\left[\begin{array}{cccc}
1 & 0 & 0 & 0 \\
0 & \cos \theta_{x 1} & -\sin \theta_{x 1} & 0 \\
0 & \sin \theta_{x 1} & \cos \theta_{x 1} & 0 \\
0 & 0 & 0 & 1
\end{array}\right] . \\
\mathbf{A u}_{\mathbf{y} 1} & =\left[\begin{array}{cccc}
\cos \theta_{y 1} & 0 & \sin \theta_{y 1} & 0 \\
0 & 1 & 0 & 0 \\
-\sin \theta_{y 1} & 0 & \cos \theta_{y 1} & 0 \\
0 & 0 & 0 & 1
\end{array}\right] . \\
\mathbf{A u}_{\mathbf{z} 1} & =\left[\begin{array}{cccc}
\cos \theta_{z 1} & -\sin \theta_{z 1} & 0 & 0 \\
\sin \theta_{z 1} & \cos \theta_{z 1} & 0 & 0 \\
0 & 0 & 1 & 0 \\
0 & 0 & 0 & 1
\end{array}\right] .
\end{aligned}
$$

- the rotation matrix on the $O_{1} X_{1} Y_{1} Z_{1}$ spatial coordinate system:

$$
A u_{1}=A u_{x 1} \cdot A u_{y 1} \cdot A u_{z 1} .
$$

- the transition matrix $O_{1} X_{1} Y_{1} Z_{1}$ coordinate system to $O_{0} X_{0} Y_{0} Z_{0}$ :

$$
\mathbf{A}_{1}^{0}=\mathbf{A t}_{1}^{0} \cdot \mathbf{A u _ { 1 } ^ { 0 }} \text {. }
$$

For body 2:

- the distance between the centers of $O_{1} X_{1} Y_{1} Z_{1}$ and $\mathrm{O}_{2} \mathrm{X}_{2} \mathrm{Y}_{2} \mathrm{Z}_{2}$ coordinate systems:

$$
\mathbf{A t}_{2}=\left[\begin{array}{cccc}
1 & 0 & 0 & l_{x 12} \\
0 & 1 & 0 & l_{y 12} \\
0 & 0 & 1 & l_{z 12} \\
0 & 0 & 0 & 1
\end{array}\right]
$$

$$
\text { - rotation around } \mathrm{O}_{2} \mathrm{X}_{2} \text { axis: }
$$

$$
\mathbf{A u}_{2}=\left[\begin{array}{cccc}
1 & 0 & 0 & 0 \\
0 & \cos \Phi_{12} & -\sin \Phi_{12} & 0 \\
0 & \sin \Phi_{12} & \cos \Phi_{12} & 0 \\
0 & 0 & 0 & 1
\end{array}\right] .
$$

The transition matrix of the $\mathrm{O}_{2} \mathrm{X}_{2} Y_{2} \mathrm{Z}_{2}$ coordinate system to $O_{1} X_{1} Y_{1} Z_{1}$ :

$$
\mathbf{A}_{2}^{1}=\mathbf{A t}_{2}^{1} \cdot \mathbf{A} \mathbf{u}_{2}
$$

The transition matrix of the $\mathrm{O}_{2} \mathrm{X}_{2} \mathrm{Y}_{2} \mathrm{Z}_{2}$ coordinate system to $O_{0} X_{0} Y_{0} Z_{0}$ :

$$
\mathbf{A}_{2}^{0}=\mathbf{A}_{1}^{0} \cdot \mathbf{A}_{2}^{\mathbf{1}}
$$

For body 3:

- the distance between the centers of $\mathrm{O}_{2} \mathrm{X}_{2} \mathrm{Y}_{2} \mathrm{Z}_{2}$ and $O_{3} X_{3} Y_{3} Z_{3}$ coordinate systems:

$$
\mathbf{A t}_{3}^{2}=\left[\begin{array}{cccc}
1 & 0 & 0 & l_{x 23} \\
0 & 1 & 0 & l_{y 23} \\
0 & 0 & 1 & l_{z 23} \\
0 & 0 & 0 & 1
\end{array}\right]
$$
the $\mathrm{O}_{3} \mathrm{Y}_{3}$ axis:

- small rotation of pendulum hemisphere around

$$
\mathbf{A} \mathbf{u}_{3}=\left[\begin{array}{cccc}
\cos \theta_{y 3} & 0 & \sin \theta_{y 3} & 0 \\
0 & 1 & 0 & 0 \\
-\sin \theta_{y 3} & 0 & \cos \theta_{y 3} & 0 \\
0 & 0 & 0 & 1
\end{array}\right]
$$

The transition matrix of the $O_{3} X_{3} Y_{3} Z_{3}$ coordinate system to $O_{1} X_{1} Y_{1} Z_{1}$ :

$$
\mathbf{A}_{3}^{2}=\mathbf{A t}_{3}^{2} \cdot \mathbf{A u}_{3}
$$

The transition matrix of the $O_{3} X_{3} Y_{3} Z_{3}$ coordinate system to $O_{0} X_{0} Y_{0} Z_{0}$ :

$$
\mathbf{A}_{3}^{0}=\mathbf{A}_{2}^{0} \cdot \mathbf{A}_{3}^{2} \text {. }
$$

The radius vector of the point $N$ on the surface of the oscillating scatterer-pendulum hemisphere in the $\mathrm{O}_{3} X_{3} Y_{3} Z_{3}$ coordinate system: 


$$
\mathbf{r}_{\mathbf{N} 3}=\left[\begin{array}{llll}
l_{x N 3} & l_{y N 3} & l_{z N 3} & 1
\end{array}\right]^{\mathrm{T}} .
$$

The position of the radius vector at $N$ point on the surface of the oscillating scatterer-pendulum hemisphere, in the $O_{0} X_{0} Y_{0} Z_{0}$ initial coordinate system:

$$
\mathbf{R}_{\mathrm{N} 3}^{0}=\mathbf{A}_{3}^{0} \cdot \mathbf{r}_{\mathrm{N} 3}
$$

By differentiation in time the expression (17) of the radius-vector provisions of the point, we get the speed of the point $N$ on the surface of the oscillating scattererpendulum hemisphere in $O_{0} X_{0} Y_{0} Z_{0}$ coordinate system:

$$
\mathbf{V}_{\mathbf{N} 3}^{\mathbf{0}}=\frac{d \mathbf{R}_{\mathrm{N} 3}^{\mathbf{0}}}{d t} .
$$

The angular velocity projection of the body 1 in the $O_{1} X_{1} Y_{1} Z_{1}$ coordinate system is determined by the formula:

$$
\omega_{11}=A u_{z 1}{ }^{\mathrm{T}} \cdot A u_{\mathrm{y} 1}{ }^{\mathrm{T}} \cdot \omega_{1 \mathrm{x} 1}+A{u_{\mathrm{z} 1}}^{\mathrm{T}} \cdot \omega_{1 \mathrm{y} 1}+\omega_{1 \mathrm{z} 1}
$$

where:

$$
\boldsymbol{\omega}_{\mathbf{1 x 1}}=\left[\begin{array}{c}
\omega_{x 1} \\
0 \\
0 \\
0
\end{array}\right] ; \boldsymbol{\omega}_{\mathbf{1 y 1}}=\left[\begin{array}{c}
0 \\
\omega_{y 1} \\
0 \\
0
\end{array}\right] ; \quad \boldsymbol{\omega}_{\mathbf{1 z 1}}=\left[\begin{array}{c}
0 \\
0 \\
\omega_{z 1} \\
0
\end{array}\right] .
$$

The angular velocity projection of the body 2 in the $\mathrm{O}_{2} \mathrm{X}_{2} \mathrm{Y}_{2} \mathrm{Z}_{2}$ coordinate system is defined as:

$$
\omega_{22}=A u_{2}{ }^{\mathrm{T}} \cdot \omega_{11}+\omega_{2 \times 2},
$$

where:

$$
\boldsymbol{\omega}_{2 \times 2}=\left[\begin{array}{c}
\omega_{x 2} \\
0 \\
0 \\
0
\end{array}\right] .
$$

The angular velocity projection of the body 3 (scatterer-pendulum hemisphere) in the $\mathrm{O}_{3} X_{3} Y_{3} Z_{3}$ coordinate system is defined as:

$$
\omega_{33}=A u_{3}{ }^{\mathrm{T}} \cdot \omega_{22}+\omega_{3 y 3},
$$

where:

$$
\boldsymbol{\omega}_{3 \mathrm{y} 3}=\left[\begin{array}{c}
0 \\
\omega_{y 3} \\
0 \\
0
\end{array}\right] \text {. }
$$

\section{Discussion}

For the calculation of the considered mechanical system the real constructive parameters of the investigated seeding opener with the oscillating scatterer of the grainfertilizer seeder have been used. In doing so, the travel speed of the seeder was $V=1,94 \mathrm{~m} / \mathrm{s}$. Coordinates of points $N_{1}, N_{2}$ and $N_{3}$ in the $O_{3} X_{3} Y_{3} Z_{3}$ coordinate systems:

$$
\begin{aligned}
& \mathbf{r}_{\mathbf{N} 1}=\left[\begin{array}{lll}
l_{N x 1} & l_{N y 1} & l_{N z 1}
\end{array}\right]^{\mathrm{T}}=\left[\begin{array}{lll}
-0,040 & 0 & -0,030
\end{array}\right]^{\mathrm{T}}, \\
& \mathbf{r}_{\mathbf{N} 2}=\left[\begin{array}{llll}
l_{N x 2} & l_{N y 2} & l_{N z 2}
\end{array}\right]^{\mathrm{T}}=\left[\begin{array}{lll}
0,040 & 0 & -0,030
\end{array}\right]^{\mathrm{T}}, \\
& \mathbf{r}_{\mathrm{N} 3}=\left[\begin{array}{llll}
l_{N x 3} & l_{N y 3} & l_{N z 3}
\end{array}\right]^{\mathrm{T}}=\left[\begin{array}{lll}
0 & 0,040 & -0,030
\end{array}\right]^{\mathrm{T}} .
\end{aligned}
$$

The considered points $N_{1}, N_{2}$ and $N_{3}$ characterize the points on the rear, front and side parts of surface of the oscillating scatterer-pendulum hemisphere respectively.

The distance between the $O_{1} X_{1} Y_{1} Z_{1}$ and $O_{2} X_{2} Y_{2} Z_{2}$ coordinate systems:

$$
\mathbf{R}_{2}^{\mathbf{1}}=\left[\begin{array}{lll}
l_{x 12} & l_{y 12} & l_{z 12}
\end{array}\right]^{\mathrm{T}}=\left[\begin{array}{lll}
-0,5 & 0 & -0,2
\end{array}\right]^{\mathrm{T}} .
$$

The distance between the $\mathrm{O}_{2} \mathrm{X}_{2} Y_{2} \mathrm{Z}_{2}$ и $\mathrm{O}_{3} X_{3} Y_{3} Z_{3}$ coordinate systems:

$$
\mathbf{R}_{\mathbf{3}}^{2}=\left[\begin{array}{lll}
l_{x 23} & l_{y 23} & l_{z 23}
\end{array}\right]^{\mathrm{T}}=\left[\begin{array}{lll}
0 & 0 & -0,03
\end{array}\right]^{\mathrm{T}} .
$$

The Figs. 2 and 3 demonstrate the trajectories of the points on the surface of the oscillating scattererpendulum hemisphere in $3 \mathrm{D}$ space and their projections on the $X O Z$ and $X O Y$ coordinate planes for the time interval of $0<t<5 \mathrm{~s}$.

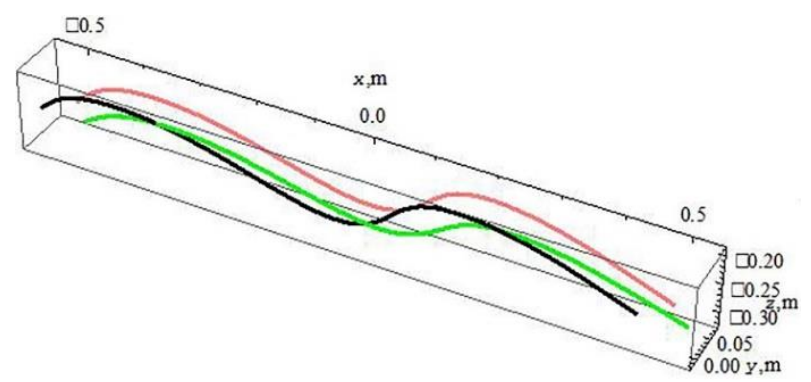

Fig. 2 The trajectory of the points $N_{1}, N_{2}$ and $N_{3}$ on the surface of the oscillating scatterer-pendulum hemisphere in $3 \mathrm{D}$ space

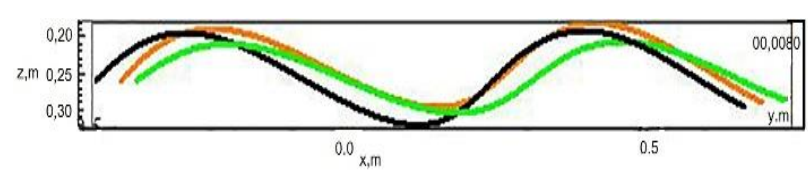

$\mathrm{a}$

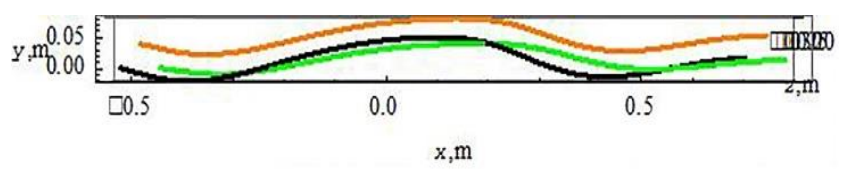

b

Fig. 3 The oscillation trajectory projections of the points $N_{1}, N_{2}$ and $N_{3}$ on the surface of the oscillating scatterer-pendulum hemisphere on the coordinate planes: $\mathrm{a}-\mathrm{XOZ} ; \mathrm{b}-\mathrm{XOY}$

From these data is seen that the considered points on the surface of the oscillating scatterer-pendulum hemi- 
sphere have different trajectories.It is revealed that the points $N_{1}$ and $N_{2}$ (red and green lines respectively) have a lengthened trajectory in comparison with the trajectory of the point $\mathrm{N}_{2}$ (black line) on the $\mathrm{XOZ}$ and $\mathrm{XOY}$ coordinate planes. The indicated allows us to state that the interaction of falling seed mass with the rear and side parts of the surface of the oscillating scatterer-pendulum hemisphere will have a positive effect on the range of their flight, consequently the spread and distribution of seeds.

There were derived the linear velocity components of the corresponding points $N_{1}, N_{2}$ and $N_{3}$ on the surface of the oscillating scatterer-pendulum hemisphere (Fig. 4).
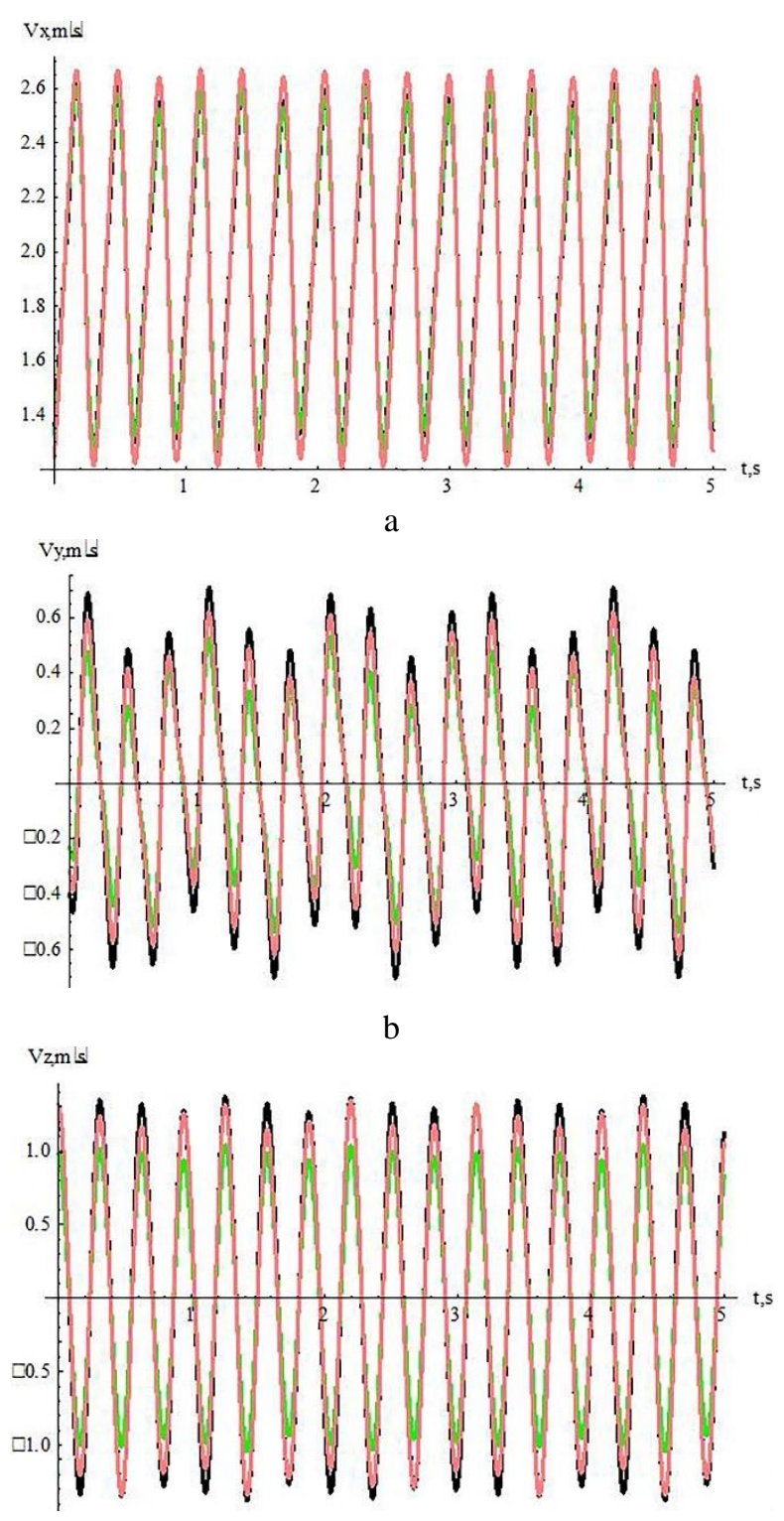

c

Fig. 4 The linear velocity components the considered points $N_{1}, N_{2}$ and $N_{3}$ on the surface of the oscillating scatterer-pendulum hemisphere: a $-V_{X} ; \mathrm{b}-V_{Y}$; $\mathrm{c}-V_{Z}$

It is revealed that oscillation amplitudes of the longitudinal component $V_{X}$ of the considered points are varied from 1.2 to $2.7 \mathrm{~m} / \mathrm{s}$, while the lateral and vertical components $V_{Y}$ and $V_{Z}$ are changed in the range of -0,6-0,6 and $-1,4-1,4 \mathrm{~m} / \mathrm{s}$ respectively. In doing so, the points $N_{1}$ and $N_{3}$ have the maximum absolute values of amplitude. The point $N_{2}$ has the minimal value of the linear velocity in all three axis. These will lead to the fact that the front and side part of the surface of the oscillating scattererpendulum hemisphere will have a greater impact force during its contact with the falling seeds, which will have a positive effect on the fright range and spread of seeds as well.

There were plotted the angular velocity components of the oscillating scatterer-pendulum hemisphere (Fig. 5).

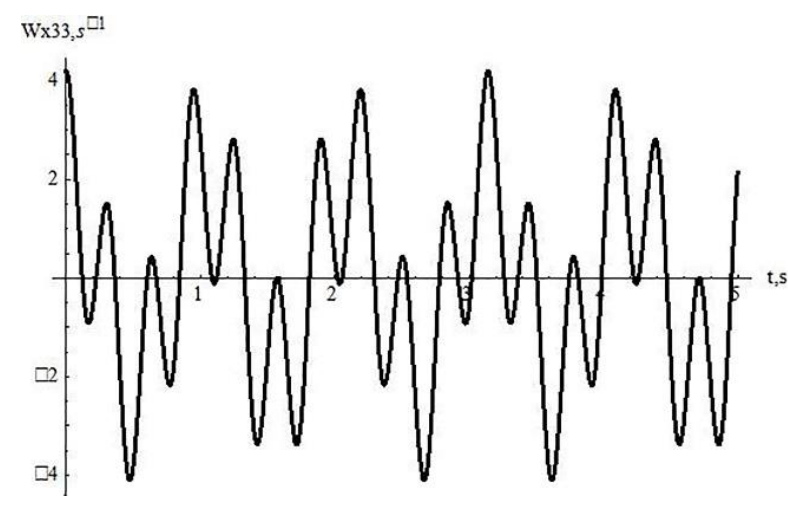

a

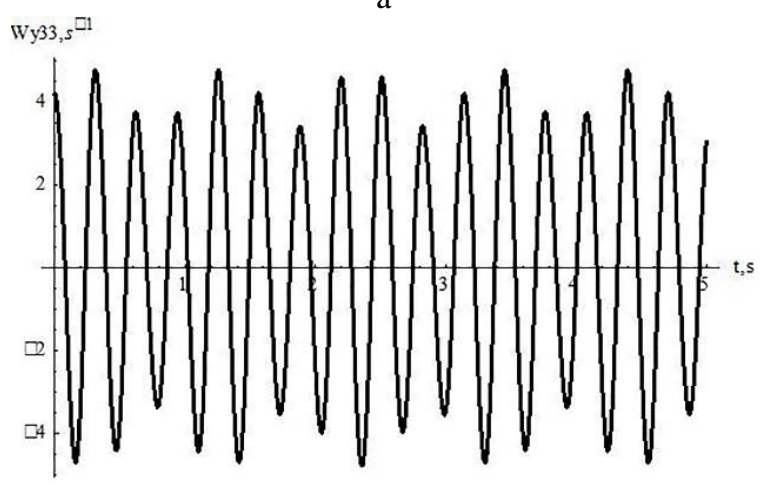

b

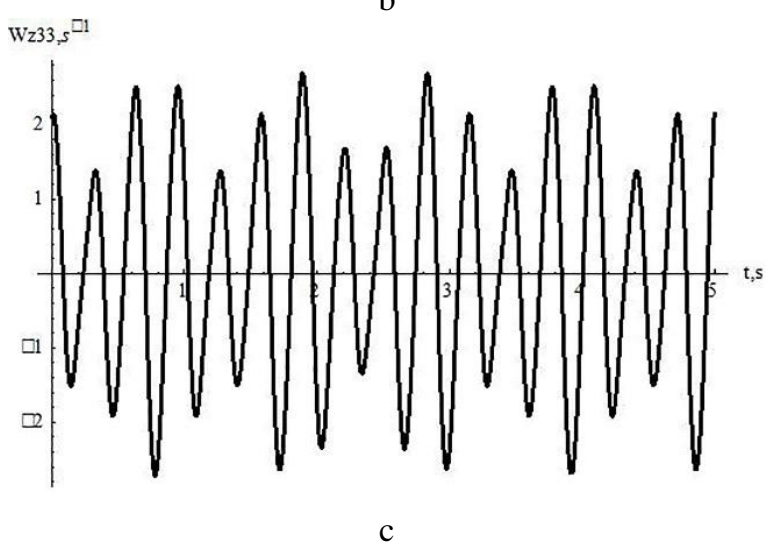

Fig. 5 The angular velocity components of the oscillating scatterer-pendulum hemisphere: $\mathrm{a}-W_{X} ; \mathrm{b}-W_{Y}$; $\mathrm{c}-W_{Z}$

It is revealed that the angular velocity components $W_{X}$ and $\mathrm{W}_{Y}$ have the maximum oscillation amplitude values. These obtained data will be used in further studies of mechanical system dynamics of the investigated seeding opener with scatterer of the grain-fertilizer seeders. 


\section{Conclusions}

1. A mathematical modelling of the developed scatterer of seeding opener of the grain-fertilizer seeder using the matrix method in $3 \mathrm{D}$ space has been created. The investigated model is presented as a mechanical system with eight degrees of freedom.

2. Based on the results of simulation, the trajectories of the points on the surface of the oscillating scatterer in the form of pendulum hemisphere in 3D space and their projections on the $X O Z$ and $X O Y$ coordinate planes are plotted. They show that the considered points on the scatterer have different trajectories, which will have a positive effect during the falling seeds - scatterer-pendulum hemisphere interaction in terms of the flight range, spread and distribution of seeds.

3 . There were derived the linear velocity components of the considered points as well as the angular velocity components of the oscillating scatterer-pendulum hemisphere. It is revealed that oscillation amplitudes of the linear and angular velocity components are varied in wide range. The indicated leads to the increase in the impact force during the contact of the falling seeds with the investigated scatterer, which will have a positive effect on the fright range and spread of seeds as well.

4. Obtained data will be used in further studies of mechanical system dynamics of the investigated seeding opener with scatterer of the grain-fertilizer seeder.

\section{References}

1. Sinyagin, I. I. 1975. Feeding area of plants, Rosselhozizdat. Moscow. 384 p. (in Russian).

2. Heege, H. J. 1981. Zur Frage der Sätechnik für Getreibe, Landtechnik 36(2): 66-69. (in German).

3. Heege, H. J.; Feldhaus, B. 2002. Control of seednumbers per unit area for grain drills, Agricultural Engineering International: the CIGR Journal of Scientific Research and Development 4: 5-11.

4. Technical support of technologies of grain crops cultivation in the system of conservation agriculture (recommendation). 2011. Kostanay department of "KazNIIMESH". 73 p. (in Russian).

5. Romanyuk, N.N.; Nukeshev, S.O.; Kakabayev, N.A.; Ageychik, V.A.; Shilo, I.N.; Toigambayev, S.K.; Eskhozhin, D.Z. 2014. Seeder: Republic of Kazakhstan patent 29217, MPK A 01B 49/04, applicant S. Seifullin Kazakh Agrotechnical University, No.29217; applied on 11.12.2013; published on 15.12.2014, Issue 12 (in Russian).

6. Shevchenko, A.P.; Domrachev, V.A. 2005. The improving of the sowing machines efficiency by optimizing structural parameters of working bodies (monograph), Omsk. 119 p. (in Russian).

7. Krasilnikov, E.V. 2009. The justification of parameters of the pneumomechanical sowing systems provid- ing uniform distribution of grain crop seeds. [PhD thesis], Omsk. 156 p. (in Russian).

8. Bespamyatnova, N.M., Lavrukhin, P.V. 1991. Investigation of the parameters and regimes of the scattered method of sowing. Equipments in Agriculture 6: 38-40. (in Russian).

9. Lavrukhin, P.V. 2000. Improvement of the process of the subsoil scattered distribution of seeds [PhD thesis], Zernograd. 159 p. (in Russian)

10. Denavit, J.; Hartenberg, R.S. 1955. A Kinamatic Notation for Lower-Pair Mechanisms Based on Matrices. ASME E, Journal of Applied Mechanics 22: 215-221.

11. Uicker, J.J. 1965. On the dynamic analysis of spatial linkage using $4 \times 4$ matrices [PhD thesis], Northwestern University, Evanston, IL.

12. Angelov, I.; Slavov V. 2006. Matrix mechanics. Kinematics. Problems. Avangard Prima. Sofia. (in Bulgarian).

13. Amiroche, F.M.L. 1993. Computer-aided design and manufacturing. Prentice Hall. Englewood Clifs. 537 p.

\section{S. Nukeshev, V. Slavov, N. Kakabayev, M. Amantayev \\ MATHEMATICAL MODELING IN 3D OF OPENER WITH SCATTERER OF THE GRAIN-FERTILIZER SEEDER}

S u m m a r y

This paper presents a mathematical modelling in $3 \mathrm{D}$ space of the scatterers of seeding opener of the grainfertilizer seeder using the matrix method. The investigated model is presented as a mechanical system with eight degrees of freedom. Based on the results of simulation, the trajectories of the points on the oscillating scatterer in the form of a pendulum hemisphere and their projections on the $X O Z$ and $X O Y$ coordinate planes as well as the linear velocity components of the considered points and the angular velocities components of the investigated scatterer are derived. The obtained data allow us to state that the use of the oscillating scatterer-pendulum hemisphere will improve the quality of sowing in terms of the flight range, spread and distribution of seeds, consequently, it will increase in the yield of crops. The results will also be used in further studies of mechanical system dynamics of the investigated seeding opener with scatterer of the grainfertilizer seeders.

Keywords: kinematics model, matrix method, scattererpendulum hemisphere, the seeding opener, the grainfertilizer seeders.

Received March 07, 2017 Accepted December 12, 2018 